\title{
Research and Experiment about Suppression of Inter Symbol Interference in the Transient Detection Theory
}

\author{
Li Zhi, a , Li Bing Yü,b \\ ${ }^{1}$ Shenyang Institute of Technology, Liaoning Province, China \\ ${ }^{2}$ University of Regina, Regina, Canada \\ alz1965@163.com, ${ }^{b}$ 997123738@QQ.com
}

\begin{abstract}
Keywords: Inter Symbol Interference; Transient Detection Theory; Suppression of Interference Abstract. The baseband signal will be influenced by the channel which bandwidth is limited with external interference, the baseband signal will generate inter symbol interference. The raised cosine FIR filter is usually used to suppress inter symbol interference in statistical detection, and it also needs strict synchronous. This paper gives out a method to suppress inter symbol interference by the transient detection, which can eliminate the external interference at same time in the process of detecting detected signal, The experiment about suppression of the inter symbol interference and the additive white noise in the transient detection theory is also set up, which can play a more effective role in suppressing the inter symbol interference.
\end{abstract}

\section{Introduction}

In addition to the influence of external and internal thermal noise interference, the digital communication system is also mainly affected by the inter symbol interference. The method of transient detection technology is not an exception, the detecting results will be impacted by the inter symbol interference, the correct judgment may not be obtained in some serious cases. The following will mainly analyze how to eliminate the inter symbol interference in transient detection.

The inter symbol interference (ISI) is caused by the channel which characteristics of transmission system (including interference and filter is not linear) is not ideal, and it lead to distortion of baseband signal in the receiving end, the waveform of the forward baseband signal will appear long tail and spread to the current code element which is sampling and detecting, so the judgment about the current code element will be impacted. When the inter symbol interference is serious, it will cause wrong judgment.

\section{Suppression of inter symbol interference in the general digital communication system}

The problem of inter symbol interference is usually discussed in the baseband transmission system, the baseband digital signal is represented by a binary " 1 " or " -1 ", but the function of the binary digital information is varied in the different digital system, the more commonly used function waveform of the positive or negative rectangular pulse representatives the information source of the binary information in the general digital communication system.

The digital information of the baseband source signal must be converted to the function waveform through the conversion circuit in the sending terminal before it is sent to the transmission channel in the digital transmission system, the distortion waveform of the source baseband digital signal must generate when it is through the limited bandwidth of the channel with the external interference. There are two kinds of interference which can impact on judgment of detection, in the first, a part frequency component of the source baseband signal will lose, which can result in the waveform distortion of the detected baseband signal at the receiving end, then the random waveform distortion of the baseband signal will generate by the channel with the random external interference. The usual solution is to design a best matched filter at the receiver for eliminating the noise and interference in the channel as far as possible, but some waveform distortion of the baseband signal must exist after processing of received signal at the receiver, so it is always necessary to set up limiter, shaper, sampler and decision 
device. The whole process of digital baseband signal transmission is summarized in Figure 1. The mathematical method is used to describe the process of digital baseband transmission based on the discussion of the block diagram.

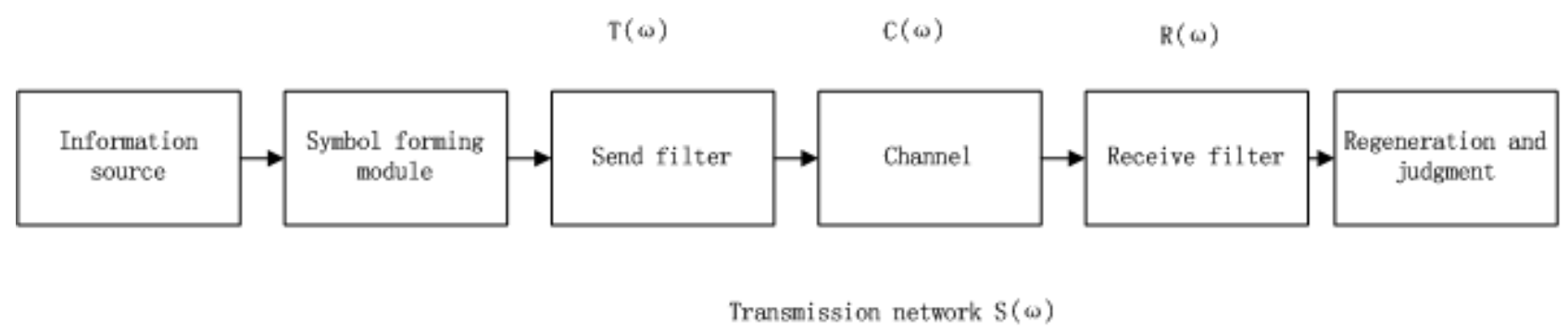

Fig.1The Model Chart of Digital Baseband Transmission System

The frequency spectrum function $S(\omega)$ is the Fourier transfer of the time-domain function $s(t)$ about transmission network in figure 1, it can be expressed as Eq1.

$$
S(\omega)=T(\omega) \cdot C(\omega) \cdot R(\omega)
$$

The whole transmission characteristic of the digital baseband transmission system can be regarded as. $S(\omega)$, The process of digital baseband signal transmission can be understood through discussing the transmission function features of a single pulse function.

The input baseband signal sequence $\mathrm{d}(\mathrm{t})$ is written as Eq2.

$$
d(t)=\sum_{-\infty}^{\infty} a_{n} g_{T}\left(t-n T_{s}\right)
$$

The Inverse transformation of $\mathbf{S}(\omega)$ is expressed as Eq3.

$$
S(t)=\frac{1}{2 \pi} \int_{-\infty}^{+\infty} S(\omega) e^{j \omega} d \omega
$$

The output signal function $\mathrm{Z}(\mathrm{t})$ of the receive filter can be obtained as $\mathrm{Eq} 4$.

$$
Z(t)=d(t) * S(t)+n(t)=\sum_{n=-\infty}^{\infty} a_{n}(t) s\left(t-n T_{s}\right)+n(t)
$$

The suppression of the inter symbol interference in digital system can be written as Eq5:

$$
Z\left(k T_{s}+t_{0}\right)=a_{k} s\left(t_{0}\right)+\sum_{n \neq k} a_{n}(t) s\left[(k-n) T_{s}+t_{0}\right]+n(t)
$$

The actual sampling value $\mathrm{Z}\left(\mathrm{kT}_{\mathrm{s}}+\mathrm{t}_{0}\right)$ not only contained the value of the source code element, but also it contained the value of the inter symbol interference and external noise. When the actual sampling value $\mathrm{Z}\left(\mathrm{kT}_{\mathrm{s}}+\mathrm{t}_{0}\right)$ is sent to the decision judgment block in the Fig 1 at the receiving end, it is difficult to judge the symbol function $\mathrm{a}_{\mathrm{k}}$ correctly in the Eq5 because of the inter symbol interference and external noise. The method to avoid error code is to ensure that the sample value correct completely without the inter symbol interference and additive noise. Raised Cosine FIR Digital Filters is widely used to suppression of the inter symbol interference in the field of statistics detection, The transfer function of the system $S(\omega)$ is the spectrum function of the received signal, it is express as Eq6.

$$
S(\omega)=\left\{\begin{array}{lr}
\frac{S_{0} T}{2}\left\{1-\sin \left[\frac{T}{2 a}\left(\omega-\frac{\pi}{T}\right)\right]\right\}, \frac{\pi(1-\alpha)}{T} \leq|\omega| \leq \frac{\pi(1+\alpha)}{T} \\
S_{0} T & 0 \leq|\omega| \leq \frac{\pi(1-\alpha)}{T} \\
0 & |\omega| \leq \frac{\pi(1+\alpha)}{T}
\end{array}\right.
$$

Here $\alpha$ is called the roll-off factor, the range of the roll off factor $\alpha$ is $0 \leq a<\infty$.

The requirement of non-inter symbol interference about the sampling value is $\alpha=1[1]$, However, the bandwidth of the method is 2 times wider as the ideal filter, only half of the baseband digital signal 
utilization efficiency can be used, so the better effect for the suppression of the inter symbol interference is obtained by the loss of bandwidth about frequency.

\section{Suppression of inter symbol interference in the transient detection}

The correct judgment of the received signal is the goal for anyone of signal detection theory, but the key is what kind of the detection method to be used. What is described above is the statistics detection method, the different statistical characteristics between the detected signal and interference is used in the signal processing, the signal has same statistical characteristics and interference has not it. The transient detection method is different from the statistics detection method, the transient characteristics between the detected signal and interference is not same too, which can be used in the suppression of inter symbol interference and additive noise

The baseband signal pulse sequence can be expressed as Eq7 in the transient detection theory.

$$
S(t)=\sum_{j=1}^{n} A_{j} g\left(t_{j}\right)
$$

The $A_{j}$ is the symbol of the detected signal code, it is +1 or -1 , the $g\left(t_{j}\right)$ is the waveform function of the detected signal code, the some detected interval of the sampling value can be written as Eq8.

$$
S(t)=S_{k}(t)+\sum_{j \neq k}^{n} S_{j}\left(t_{j}\right)
$$

The $S_{k}(t)$ is the sampling value which is being detected, the second items is the part of inter symbol interference, which should be eliminated. It also can be regarded as part of the external interference and be written as Eq9 in the transient detection principle, so the inter symbol interference can be eliminated with the same detected method of the external interference.

$$
N(t)=n(t)+\sum_{j \neq k}^{n} S_{j}\left(t_{j}\right)
$$

The expression of mixed signal which received at the receiving end can be written as Eq10.

$$
E(t)=S_{k}(t)+N(t)=S_{k}(t)+n(t)+\sum_{j \neq k}^{n} S_{j}\left(t_{j}\right)
$$

The interference differential equation of the transient detection and its initial value can be obtained through the Eq9 and Eq10 and written as Eq11[2]..

$$
\sum_{j=0}^{K} \phi_{j}(t) \cdot N^{(j)}(t)=V(t) \quad N^{(j)}(0)=\mu_{j,} j=0,1,2 \ldots \ldots k-1
$$

The characteristics of the code waveform which is designed in the transient detection theory has a tail, the tail can be reduced to negligible if the attenuation parameters is selected appropriately, the inter symbol interference can be eliminated according to the transient detection equation as Eq12.

$$
S\left(t_{j}\right)=E\left(t_{j}\right)-N\left(t_{j}\right)
$$

\section{The design about the experiment on suppression of the inter symbol interference in transient detection theory}

(1)The baseband digital communication system about suppression of the inter symbol interference. This experiment mainly verifies the effectiveness about suppression of the inter symbol interference in transient detection theory. Therefore, the basic baseband digital baseband communication system is designed as the flow chart Fig2.

In order to overcome the uncertainty about suppression of the inter symbol interference in the experiment, the experimental transmission channel is assumed an ideal channel with the additive white noise. If the adaptive equalization techniques are used in the receiver, the effective of suppression of the inter symbol interference will be better. 


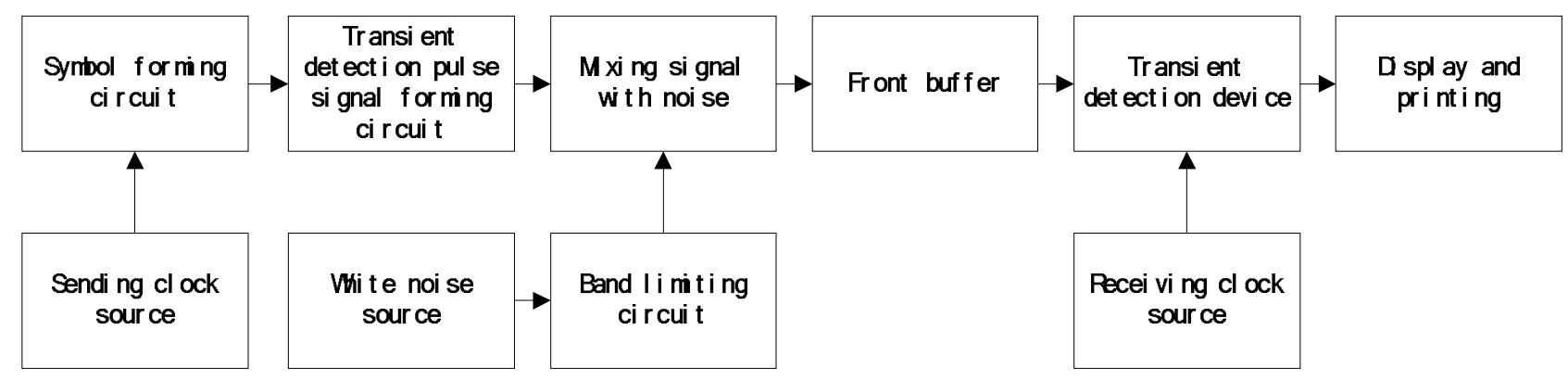

Fig2 The experiment block diagram about suppression of the inter symbol interference

(2) Experimental results of suppression of the inter symbol interference. The experimental results of the suppression of the inter symbol interference are shown in the Table 1.

Table 1 The Result about supressing of the inter-symbol interference in transient detection experiment

\begin{tabular}{clccc}
\hline No. & $\mathrm{SNR} / \mathrm{dB}$ & $\mathrm{Pe}(\mathrm{BN}=1 \mathrm{kHz})$ & $\mathrm{Pe}(\mathrm{BN}=2.2 \mathrm{kHz})$ & $\mathrm{Pe}(\mathrm{BN}=3.4 \mathrm{kHz})$ \\
\hline 1 & -26.0206 & 0.1720253 & 0.1940355 & 0.2125902 \\
2 & -23.25455 & $7.316707 \mathrm{E}-02$ & 0.1153846 & 0.1229718 \\
3 & -21.15984 & $3.102464 \mathrm{E}-02$ & $5.791767 \mathrm{E}-02$ & $5.919472 \mathrm{E}-02$ \\
4 & -19.47342 & $8.188101 \mathrm{E}-03$ & $1.983173 \mathrm{E}-02$ & 0.029372 \\
5 & -18.0618 & $1.878005 \mathrm{E}-03$ & $6.234976 \mathrm{E}-03$ & $8.939304 \mathrm{E}-03$ \\
6 & -16.84784 & $1.126803 \mathrm{E}-03$ & $3.004808 \mathrm{E}-03$ & $4.131611 \mathrm{E}-03$ \\
7 & -1578293 & 0 & $6.009614 \mathrm{E}-04$ & $2.328726 \mathrm{E}-03$ \\
8 & -14.83444 & 0 & 0 & $6.009616 \mathrm{E}-04$ \\
9 & -13.9794 & 0 & 0 & $6.253606 \mathrm{E}-04$ \\
10 & -13.20104 & 0 & 0 & 0
\end{tabular}

The code sending rate of experimental parameters is $2.5 \mathrm{kbps}$ in the Table 1 , the bandwidth of the band limiting circuit is set to $1 \mathrm{kHz}, 2.2 \mathrm{kHz}$ and $3.4 \mathrm{kHz}$, the signal bandwidth of the signal is 1.34 $\mathrm{kHz}$.

\section{Conclusions}

The following conclusions can be drawn through the results of Table 1, the transient detection theory not only is outstanding in suppression of the inter symbol interference, but also in eliminating the external additive noise which bandwidth is limited and the interference of the tail[3] of the transient detection symbol. It is entirely different method between the statistical detection and the transient detection. Therefore, the high speed data transmission can be achieved in a narrow bandwidth channel by transient detection theory.

\section{References}

[1] Fangchangxin,Caolina: Principle of communication(the seventh version), edit by National Defence Industry Press(2012)

[2] Lizhi. A new method of detecting the weak deterministic signal from strong additive noise. ICCSIT2010. Volume 7(2010), p. 352,..

[3] Lizhi. The design of no distortion filter based on transient detection. ICCSIT2010. Volume 7(2010), p. 349 